\title{
5G: Future Mobile Technology-Vision 2020
}

\author{
Saurabh Patel \\ EC Department \\ NSIT,Jetalpur \\ Gujarat,India
}

\author{
Malhar Chauhan \\ EC Department \\ NSIT,Jetalpur \\ Gujarat,India
}

\author{
Kinjal Kapadiya \\ EC Department \\ NSIT,Jetalpur \\ Gujarat,India
}

\begin{abstract}
Currently, Mobile operators are busy with deployment of 4G technology namely, LTE-advanced or WIMAX $802.16 \mathrm{~m}$. This $4 \mathrm{G}$ technology will be concluded within two years. 5G technology is not standardizing yet, probably $5 \mathrm{G}$ standard will define in two to three years, and its deployment will start around 2020. In future, people will expect same quality of internet connectivity as the device is capable. This technology will include all types of advanced features, which make 5G technology more powerful. The main features we want to add in $5 \mathrm{G}$ mobile network is that user can simultaneously connect to the multiple wireless technologies and can switch between them. Forthcoming mobile technology has to support IPv6 and flat IP. This paper explains different technology which we want to include making future mobile technology more powerful and more in demand.
\end{abstract}

\section{Keywords}

5G, Future Mobile Technology, IP, Mobile Networks

\section{INTRODUCTION}

It's at the moment just over last few years since the smart phone changed the world of the mobile Internet and mobile connectivity is changing the way we live and work. Now a day Smart-phone selling is more compared to mobile phone selling, it indicates that technology is changing. With the advent of tablets, the online media consumption patterns of users are going to change very fast. Now a day's more and more people start purchasing their products with tablet and mobile phone. That means more and more people use phones to read consumer reviews, compare prices and view photos of product. People expect to have the same speeds on their tablet PC and in a smart phone as they get at the desktop at home. Traffic on wireless networks has been doubling annually and around 2020, data consumption will be thirty times more. Current 4G technology will not be capable of carry this rapid increase of data consumption.

In reality, $5 \mathrm{G}$ does not exist yet but the future network next to $4 \mathrm{G}$ we say as $5 \mathrm{G}$. So far, we heard about only LTE advanced, which give peak download speed of $1 \mathrm{Gbps}$ and upload speed of $512 \mathrm{Mbps}$, but you cannot drain its full capacity with your mobile devise. It is expected that $4 \mathrm{G}$ standard will be concluded within two years. 5G network will not be only more speed but also capable of carry more data. This generation is expected to be rolled out around 2020. We know that frequency is a finite resource. In $5 \mathrm{G}$, network might solve the problem of frequency licensing and spectrum management issues. The $5 \mathrm{G}$ terminals might have software-defined radios also it has different modulation schemes and error-control schemes. It provides hundreds of channel without streaming.
Table 1 Comparison of 3G, 4G and 5G Technology

\begin{tabular}{|c|c|c|c|}
\hline $\begin{array}{l}\text { Technology/ } \\
\text { Features }\end{array}$ & 3G & 4G & $5 G$ \\
\hline $\begin{array}{l}\text { Data } \\
\text { Bandwidth }\end{array}$ & 2Mbps & $\begin{array}{l}2 \mathrm{Mbps} \text { to } \\
1 \mathrm{Gbps}\end{array}$ & $\begin{array}{l}\text { 1Gbps \& } \\
\text { Higher }\end{array}$ \\
\hline Standards & $\begin{array}{l}\text { WCDMA } \\
\text { CDMA- } \\
2000\end{array}$ & $\begin{array}{l}\text { Single } \\
\text { unified } \\
\text { standard }\end{array}$ & $\begin{array}{l}\text { Single } \\
\text { unified } \\
\text { standard }\end{array}$ \\
\hline Technology & $\begin{array}{l}\text { Broad band- } \\
\text { width } \\
\text { CDMA, IP } \\
\text { technology }\end{array}$ & $\begin{array}{l}\text { Unified IP } \\
\text { and seamless } \\
\text { combination } \\
\text { of broadband, } \\
\text { LAN/WAN/ } \\
\text { PAN and } \\
\text { WLAN }\end{array}$ & $\begin{array}{l}\text { Unified IP } \\
\text { and seamless } \\
\text { combination } \\
\text { of broadband, } \\
\text { LAN/WAN/P } \\
\text { AN/WLAN } \\
\text { and wwww }\end{array}$ \\
\hline Service & $\begin{array}{l}\text { Integrated } \\
\text { high quality } \\
\text { audio, video } \\
\text { and data }\end{array}$ & $\begin{array}{l}\text { Dynamic } \\
\text { information } \\
\text { access, wear- } \\
\text { able devices }\end{array}$ & $\begin{array}{l}\text { Dynamic } \\
\text { information } \\
\text { access, wear- } \\
\text { able devices } \\
\text { with AI } \\
\text { capabilities }\end{array}$ \\
\hline $\begin{array}{l}\text { Multiple } \\
\text { Access }\end{array}$ & CDMA & CDMA & $\begin{array}{l}\text { CDMA \& } \\
\text { BDMA }\end{array}$ \\
\hline $\begin{array}{l}\text { Core } \\
\text { Network }\end{array}$ & $\begin{array}{l}\text { PACKET } \\
\text { NETWORK }\end{array}$ & INTERNET & INTERNET \\
\hline Handoff & Horizontal & $\begin{array}{l}\text { Horizontal \& } \\
\text { Vertical }\end{array}$ & $\begin{array}{l}\text { Horizontal \& } \\
\text { Vertical }\end{array}$ \\
\hline
\end{tabular}

Next Figure Shows the Evolution and mobile technologies over last two decade.

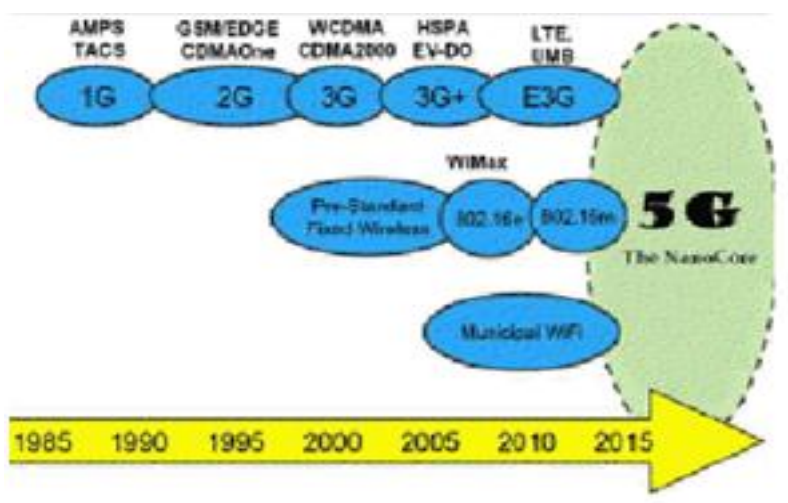

Fig. 1: Evolution of Mobile Technologies 


\section{FUTURE MOBILE TECHNOGOLIES}

\subsection{Cognitive Radio or Software Define Radio}

Because of a steady increase the communication speed and number of user requirements of a radio spectrum also increase. This lead to initiate to make more efficient use of the spectrum which gives cost-effective results. Cognitive radio technology will be more efficient radio communications systems to be developed. This new radio technologies share the same spectrum efficiently by finding unused spectrum and adapting the transmission scheme to the requirements of the technologies currently sharing the spectrum. Cognitive radio will have knowledge of free channel and occupy channel, type of data to be transmitted, modulation scheme, position of receiving equipment and also aware of the environment. With the knowledge of above parameters radio should capture the best available spectrum to meet user requirements and quality of services. Cognitive radio should be software defined radio so it can be fully reconfigurable based on above parameters and user demand.

when the level of occupancy increase then cognitive radio systems has to move continuously from one channel to another this will reduces the efficiency of system and in the worst case, system will be inoperable. As a use of cognitive radio increase in a single frequency then signal will appear on new frequency continuously so that effective algorithm must be develop so that CR system will move only when it is necessary.

Cognitive Radio Spectrum Sensing techniques are given below.

\subsubsection{Continuous Spectrum Sensing}

In this system spectrum occupancy will monitor continuously, and cognitive radio system will use the spectrum on a noninterference basis for the user.

\subsubsection{Monitor for Empty Alternative Spectrum}

When primary user return to the spectrum then cognitive radio system must have an alternative spectrum available so it can switch secondary user on it.

\subsubsection{Monitor type of transmission}

The cognitive radio must have knowledge of transmission used by users so that interference can be ignored

\subsection{Beam Division Multiple Access}

Mobile communication system has to provide good quality service to a large number of users at lower cost. Big challenge for wireless technology is to increase system capacity and quality within a limited frequency spectrum. For solving this problem multiple access techniques are required. Now days in the wireless system FDMA, TDMA, CDMA and OFDM multiple access techniques are used. In present multiple access technique's frequency and time are divided among multiple users. Therefore, capacity of a mobile communication system depends on time and frequency. Korean research and development has suggested BDMA as a radio interface for $5 \mathrm{G}$, which is not depended on frequency/time resources.

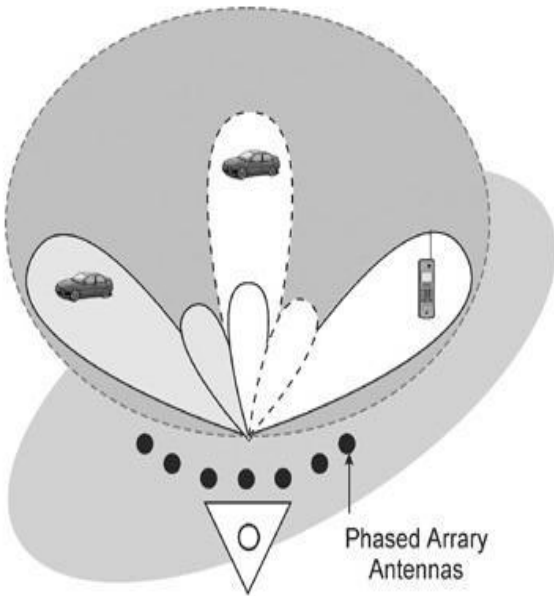

Fig. 2: Beam Division Multiple Access

In BDMA technique base station allocates separate beam to each mobile station for that it divides the antenna beam according to the location of mobile stations. This multiple access technique significantly increases the capacity of the system. Initially, base station and mobile station don't know each other positions so mobile station finds its position and moving speed transmit it omnidirectionally thereof to the base station. Based on moving speed and position of mobile station, the base station calculates a direction and width of a downlink beam. When mobile station and base station are in LOS state and know the position of each other then both can communicate with each other by the separate beam. In BDMA technique, multiple beams of distinct patterns are formed using the phase array antenna. When mobile stations are located at a different angle with base station then base station transmits the different beam at the different angle to transmit data simultaneously. If mobile stations are at the same angle with base station, after that they share same beam. The base station can change the width of beams, number of beam and direction according to a communication environment. The beam is three dimensionally divided so it can accommodate more users. The radiation efficiency of antenna can be maximized with matching of radiation pattern of mobile station and base station antennas. Mobile stations are at the same angle with base station share same beam which lower MCS level or peak to average power ratio problems.

\subsection{Support IPv6}

In the 5G system, each cell phone will have permanent "Home" IP address and "care of address" which represents its actual location. When a computer on the Internet wants to communicate with cell phone after that first, it sends a packet to the home address and subsequently server on home address send a packet to the actual location through the tunnel. Server also sends a packet to the computer to inform the correct address so that future packets will send on that address. Because of multiple layer of subnetting and many addresses IPv6 is needed for mobility. IPv6 addresses are 128 bit, which is four times more than 32 bit IPv4 address. This 128 bit address will be divided into four parts. The first 32-bit part may be defined as the home address of a device, second part may use for care of address, Third part for tunnelling to establish a connection between wire-line and wireless network and last part of IPv6 address may be used for VPN sharing. The goal of 5G network is to replace current core mobile network with a single worldwide network standard based on IPv6 for control, packet data, video and voice. This will give 
uniform voice, video and data services to the mobile host based on IPv6.

\subsection{Support Flat IP}

Flat IP network is the key feature to make $5 \mathrm{G}$ acceptable for all kinds of technologies. In next generation of network, it is beneficial to transmit all voice, video and data using packet switching instead of circuit switching. Each mobile devises have to allocate IP based on connected network and its location.

In flat IP architecture devises are identified using the symbolic name instead of conventional IP format. The term 'flat' is used to emphasize that data is no longer routed by traversing a hierarchy from originating user through multiple layers of aggregation to a central core and then re-routed back out in a multilayer dis-aggregation hierarchy to the targeted user. Instead, the flat core routers of the originating user routes' data directly to the local flat core router of the target user. This requires direct connections, or failing that requires shortest path connections between flat core routers. In this technology only one access specific node type is available, and it will be integrated in BTS is IP capable. This technology reduces the number of component lower the operation cost and investment. Because of fewer central components to disrupt the system give low failure rate and lower latency

With above advantages of IP, the negative side is security challenges. The internet is open not only for developers but also for criminals and hackers. The Internet brings us not only voice and video but also virus, Trojan horses and phishing attack, which is challenges for future mobile networks.

\subsection{Multihoming}

It is a technique used to increase the reliability of the Internet connection for an IP network. Next generation of network will support vertical handover, and user can simultaneously be connected to several wireless access technologies and move between them. The internet through multiple network interface or IP addressable to a single device is called multihoming. For multihoming network configuration assign multiple IP address to different wireless technology available on a same device. If one of the link fails, then its IP address will be unreachable but other IP address will still work so we can access the internet.

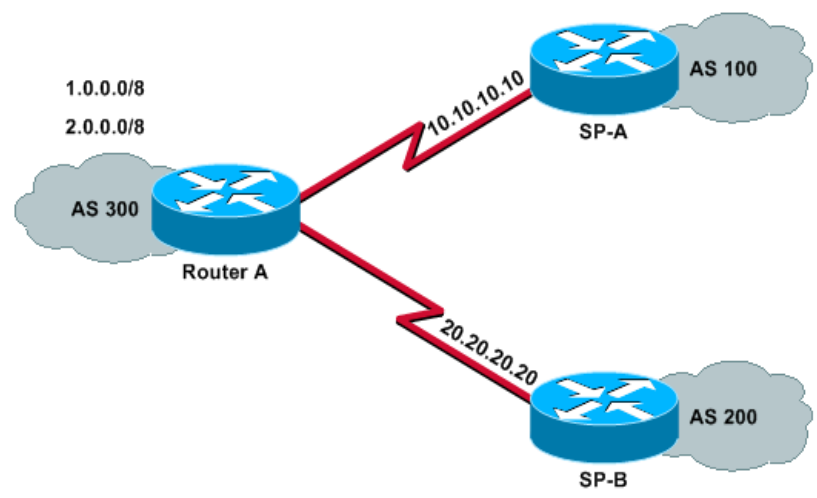

Fig. 3: Multihoming

Above figure shows the BGP network for two service provider. This way techniques use to increase network reliability. Multihoming is increased in popularity because of IPv6 address availability, support more network protocol for multihoming than traditional IPv4 address. IPv6 address has availability of provider independent address space. This technique gives work like IPv4, support traffic balance across multiple providers and maintains existing TCP and UDP sessions through cut-over's.

\subsection{Pervasive Networks}

It provides ubiquitous computing. The growing demand for mobile broadband technology also increases air interference technology and provides local area connectivity to the wide area. Tomorrow network will be "network of networks" will provide uninterrupted service when roaming across many radio access schemes. The user can simultaneously be connected to several wireless access technologies and move between them. These access technologies can be a $2.5 \mathrm{G}, 3 \mathrm{G}$, $4 \mathrm{G}$, or $5 \mathrm{G}$ mobile networks, Wi-Fi, WPAN, or any other future access technology. If the user is getting multiple streams for from different sources and one of these sources fail, then other technology provides the connection. In 5G user can provide multiple concurrent data transfer and also user can move globally. Beyond 4G, network gives media independent handover, vertical handover and IEEE 802.21 support.

IEEE standard 802.21 supports handover between same type of networks as well as distinct type of networks. This standard allows handover to and from 802.3, 802.11, 802.15, 802.16, $3 \mathrm{GPP}$ and $3 \mathrm{GPP} 2$ networks with different handover mechanism. This type of handover is called vertical handover. Mobile IP provides vertical handover mechanisms for different types of networks, but can be slow in the process. To support vertical handover mobile station must have dual mode cards so it can work on WLAN and UMTS band and modulation scheme. In cellular networks, handover decision is made on relative signal strength, block call and call drop rates, etc.. In vertical handover decision is taken based on relative signal strength, user preference, application types, network conditions, cost, etc...

\subsection{Group Cooperative Relay Techniques}

With the development of the MIMO (multiple input multiple output) system gives higher throughput and reliability in a wireless network. This technology is clearly advantageous for base station side but not on the user side due to size and power consumption. The alternate way for this is Group Cooperative diversity techniques. In cooperative communication, source transmits data to the destination at that time neighbor user (Relay user) can also hear a transmission. The relay user also processed and forwards this message to the destination and received signals are combined. Both Signals are transmitted with the different path gives diversity. Mostly, Cooperative diversity can be performed two ways.

\subsubsection{Amplify-and-Forward}

In this relay station receive the signal amplify it and then forward it without decoding. This system is useful when relay node has less power. Major drawback of this system is it also amplifies noise at relay node.

\subsubsection{Decode-and-Forward}

In this relay station receives the signal decode it then forward it to the destination. We can add error correcting code at relay node. This is only possible if relay station has enough power. 


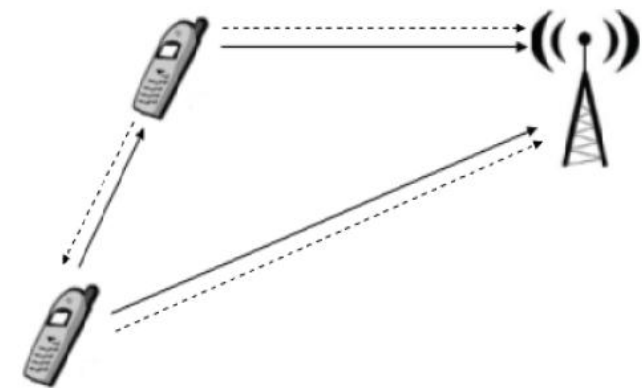

Fig. 4: Group Cooperative Communication

\subsection{Mobile Cloud Computing Support}

Cloud computing is new and unique technique to access data like documents, application, video files, music file, etc. from any place without carrying any data-storage devices. By having all the information on cloud user can access all data from anywhere in the world any time. Best example of cloud computing is Gmail. In this service, all mails are stored on Gmail server, and also all processes are done on cloud. Users give the command then process occurs on Gmail's server, and result is displayed on a screen.

Next figure shows the cloud computing .Mobile world is depended upon two factors one network availability $(2 \mathrm{G}, 3 \mathrm{G}$, $4 \mathrm{G}$ WIFI, etc.,) and second is handset availability (Feature phones, Smart Phones). Now days there is more user carry "feature" mobile phones instead of smart phones, and they want all features in their phone what we see in the smart phone. Because of low processing capability, low data storage and less power mobile cloud computing is best option. It will allow to feature mobile phone users having same data access like smart phone difference is that feature phone data will not physically be stored on the phone. This advantage of cloud computing allows the mobile operator to target a larger market instead of only smart phone users.

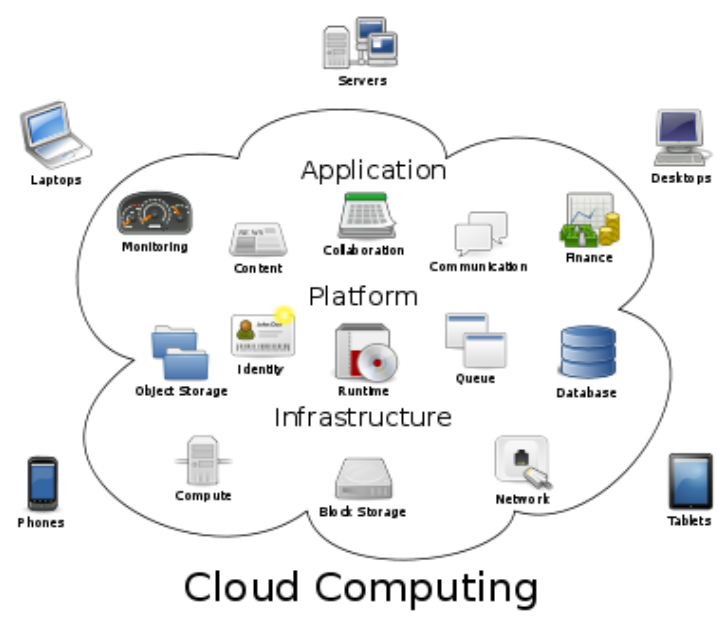

Fig. 5: Cloud Computing

Another reason for mobile cloud computing is due to mobile applications. . Mobile applications provide a number of benefits to the user, but we always find the problem is some applications work on the specific platform known as a mobile operating system. For example, one mobile application can run on Bada phone but can't run on blackberry. This problem can be solved with the help of cloud computing. In this, the application runs on the specific device called cloud, and user can access the data and application. Requirement is user must have the internet not to have more computing capacity.

\subsection{High Altitude Stratospheric Platform Station Systems}

As a demand of data grows continuously to transfer high data require large band width. To use high-bandwidth solution is to use lower wavelength waves, which require line of sight propagation, which is challenged to compare with lower frequency propagation. Wireless communication services are provided by terrestrial and satellite systems. The terrestrial systems are used to provide services in complex propagation areas. Satellite links are used when terrestrial links are not available. Now a day's research is going on aerial platforms at high altitudes provide line of sight propagation.

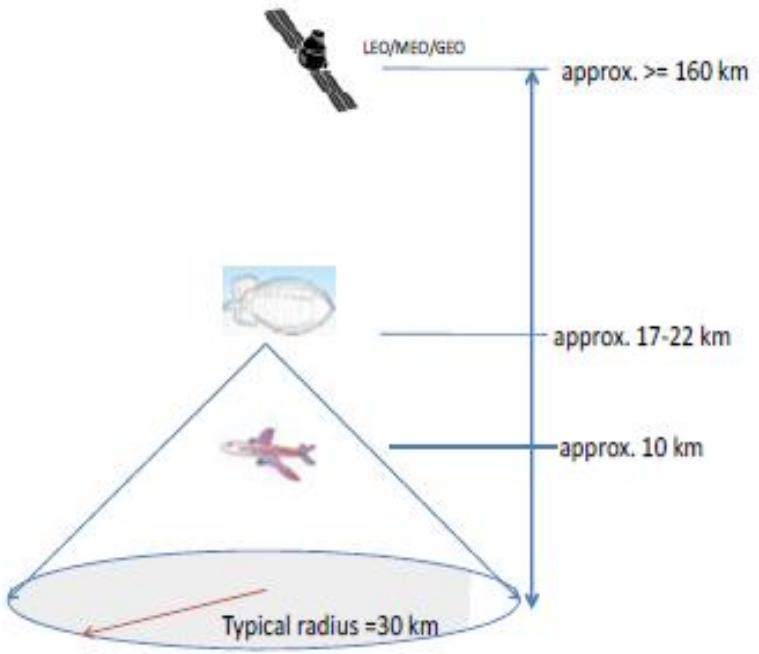

Fig. 6: HAP System above 17 22km.

A high-altitude platform can be airplane or balloon designed to operate at very high-altitude approx. $17-22 \mathrm{~km}$ and is able to stay there for long time. HAP is powered by battery, engine or solar cell. HAPs work as a base station can compare with tall antenna delivers a wireless communication. It is good techniques to serve broadband wireless service. HAPs give a coverage radius of $30 \mathrm{Km}$ so we can establish single HAP instead of several terrestrial base stations in suburban and rural areas. HPA does not require expensive launching like satellite which gives cost-effective way. HAP can easily deploy in hours so it is used in emergency or in disaster. HAPs offer the line of sight links gives high capacity of broadband applications.

Because of the wind, HPAs will vary in position vertically and laterally. This movement changes the look angle from the ground terminal. If this variation is greater than beam width of an antenna, then it requires the gain to operate the link.

\subsection{Less Power Consumption}

With increasing bit rates led to increased energy consumption in base satiations. Main challenge for future mobile networks is to reduce power consumption. In cellular networks base station consume more than $60 \%$ of the power so it is preferable to reduce power consumption in base station elements. Currently, networks are designed with the consideration of peak load not with medium and low load consideration. In real scenario if the load on a network increased, then coverage will decrease and load decrease, then coverage increase. To reduce the power consumption network 
topology will design in such a way that as load on decrease base station starts to cover more regions, and we can shutdown some of the base stations.

\section{5G Super Core Architecture}

The Newer Generations from $2 \mathrm{G}$ to $5 \mathrm{G}$ were always identified by increasing bit rates. As the $4 \mathrm{G}$ network provides adequate RF coverage and capacity for high-volume data applications and lower latency for voice applications. Beyond 4G, there will no need for newer technology and wireless standards. This newer technology will integrate network technologies rather than expansion of older technology. New network applications will be developed to integrate various engineering practices as health care, mechanical, chemical, banking, etc.
Existing telecom networks are in hierarchical ways, where traffic is aggregated at BSC/RNC and then routed to gateways. Flat IP architecture will reduce the burden on aggregation point, and traffic will directly move from base station to the media gateways. When transmission from TDM, ATM platforms to flat network a common ALL IP network will be emerged. Super core is based on IP platform. All network operators can be connected to one super core with massive capacity. Each engineering practice has their own standards. To integrate these standards requires the systematic and time-consuming process. One common architecture is required, which creates a common platform for all engineering practices to regularize the inter connectivity issue as well as knowledge sharing.

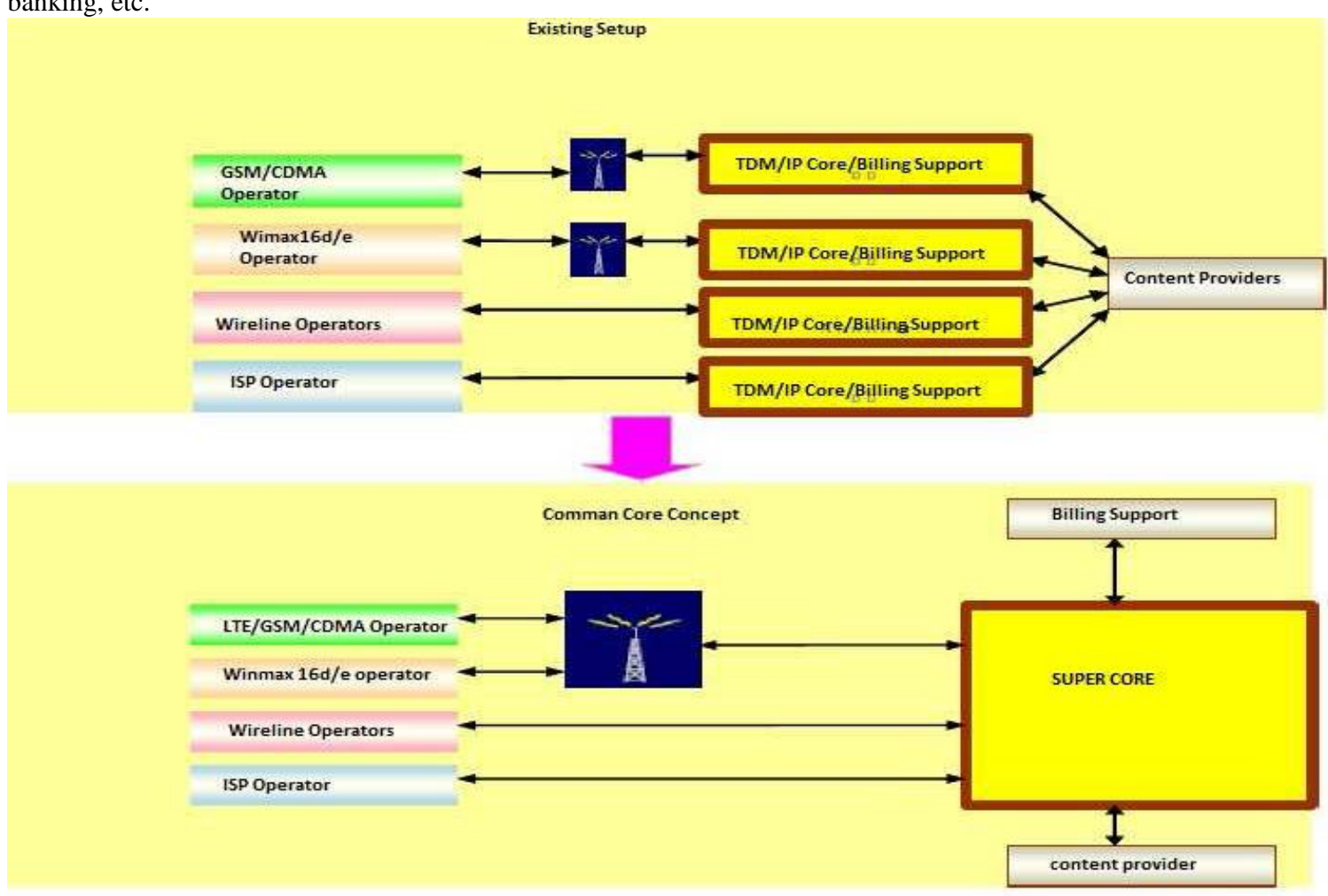

Fig 7: Super Core Architecture

\section{CONCLUSION}

The 5G mobile technology will be implemented at the end of the current decade. In this paper, we explain different mobile technology we want to include in a future mobile network. This mobile technology will offer high data rate, efficient and reliable communication at an affordable rate. Basically this paper shows the technology can be added in the $5 \mathrm{G}$ so by this Add on the future technology can become more efficient and helpful for society.

\section{REFERENCES}

[1] A. Mahmood, "Cooperative Diversity in Wireless Networks", Journal of Computer Science and Engineering, pp. 184-187, Review 3 (1) (2010).

[2] T. C. Tozer and D. Grace, "High-altitude plateforms for wireless Communications", Electronics and communication Engineering Journal, June 2001

[3] Shakil Akhtar," Evolution of Technologies, Standards, and Deployment of 2G-5G Networks", White papers, Clyton state university, August 1,2008
[4] Imthiyaz Ali, "Beam division multiple access (BDMA) for 5g",Next Generation ,April 3,2011

[5] Tejas Dave, "Emergence Of Mobile Cloud Computing", Tracking Developments In Indian Telecom Industry, May, 2011, pp. 1-10

[6] Flat IP Available: http://en.wikipedia.org/wiki/Flat_IP

[7] Peter Sayer,"Flat IP Mobile Network Face New security challenges",IDG News Service, October 10,2007

[8] Cognitive Radio Available: http://www.radioelectronics.com/info/rf-technology-design/cognitiveradio-cr/technology-tutorial.php

[9] Bradley Mitchell,"Multihoming",About.com Guide

[10] Multihoming http://en.wikipedia.org/wiki/Multihoming
Available: 